\title{
Effect of Strain Rate on Plastic Deformation Behavior of Y-CSZ Single Crystal
}

\author{
Deock-Soo Cheong and Chang Sam Kim*i \\ Department of Materials Science and Engineering, Dankook University, Cheonan, Chungnam 330-714, Korea \\ *Battery Research Center, Korea Institute of Science and Technology, Seoul 136-791, Korea
}

(Received November 9, 2009 : Received in revised form December 10, 2009 : Accepted December 10, 2009)

\begin{abstract}
Yttria stabilized zirconia (Y-CSZ) single crystals show plastic deformation at high temperatures by activating dislocations. The effect of strain rate on the plastic behavior of this crystal was studied. As increasing strain rate from $\varepsilon=1.04 \times 10^{-5} \mathrm{sec}^{-1}$ to $2.08 \times 10^{-5} \mathrm{sec}^{-1}$ the yield drop was suppressed and resulted in higher Young's modulus and yield stress. Dislocation structures of the strained crystals were analyzed using a transmission electron microscope to elucidate the plastic behavior of these crystals. In the early stage of plastic deformation, dislocation dipoles and prismatic dislocation loops were formed in both samples. However, dislocation density was increased by increasing strain rate. Strong sessile dislocations were observed in the sample with higher strain rate, which may cause the higher work hardening.
\end{abstract}

Key words yttria stabilized zirconia, plastic deformation, dislocations, yield stress, strain rates.

\section{Introduction}

Zirconia has been known as oxide with high index of reflection and diffusivity of anions. With these properties, it has been applied for jewelry industry and oxygen sensors. Y-CSZ(Yttria Fully Stabilized Zirconia) single crystal were deformed by dislocation movement during high temperature deformation which showed yield drop by the activation of secondary slip system. ${ }^{1,2)}$ And yield drop was occurred after the yield point by activating the secondary slip system when sample was deformed along [112]. The dislocation structures in this crystal were studied comprehensively by Cheong at. al. ${ }^{3)}$ The yield drop can be occurred when dislocation movement in the sample were faster than cross head speed. ${ }^{4)}$ When cross head speed was increased, plastic behavior of this crystal was changed by increasing yield stress and suppressing the yield drop.

We investigated the effect of strain rate on the early stage of plastic deformation of this crystal by adopting compression tests at high temperatures and an analysis of dislocation substructures with transmission electron microscopy (TEM).

\section{Experimental Procedure}

\section{Preparation of compression sample}

Y-CSZ single crystals were received from Ceres Company

Corresponding author

E-Mail : cskim@kist.re.kr (C. S. Kim)
(Waltham, MA) which were doped with $9.4 \mathrm{~mol} \% \mathrm{Y}_{2} \mathrm{O}_{3}$ grown by skull melting process. The crystals were rod shape, about $40 \mathrm{~mm}$ in diameter and $100 \mathrm{~mm}$ in length. The orientation were determined by Laue X-ray back reflection. The geometry and orientation of the specimen were shown in Fig. 1. Three X-Ray Laue photographs show the orientation of the sample. And the size of sample was $3 \times 3 \times 8 \mathrm{~mm}^{3}$.

\subsection{Deformation at high temperature}

Plastic deformation experiments were carried out in a screw-driven Instron machine equipped with compression

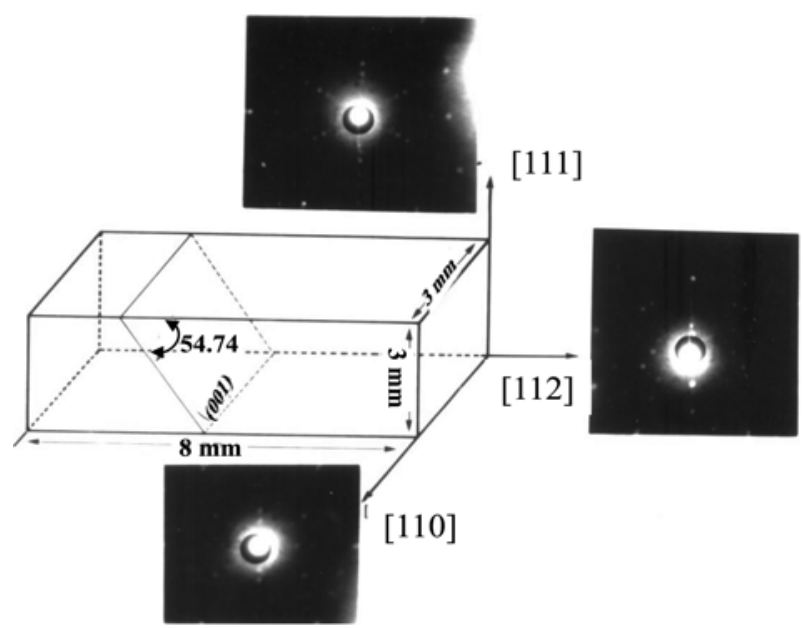

Fig. 1. The geometry and crystallographic orientation of the compression sample. Three Laue pictures show the orientation of the sample and the primary slip plane, $\{001\}$ was also illustrated in this figure. 


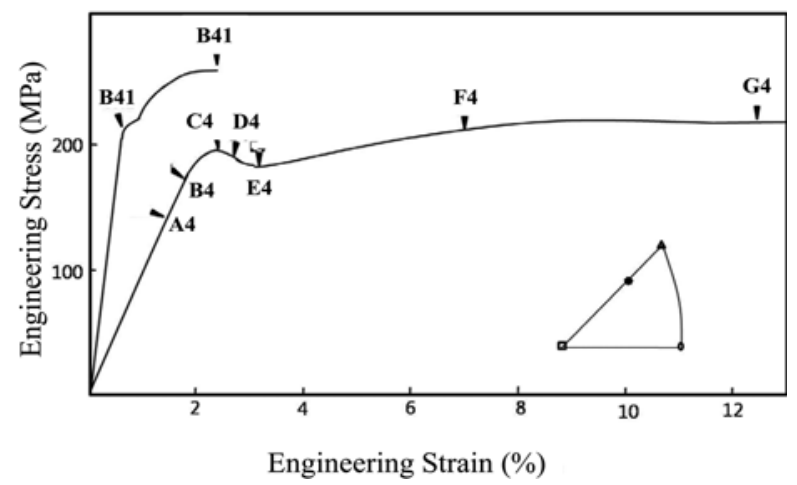

Fig. 2. Stress-Strain curve of the sample deformed at two different strain rates. The orientation and temperatures were same for both samples. Stereographic triangle showed the compression direction.

apparatus, consisting of deformation rods surrounded by high temperature furnace. The cross head speeds of the compression tests were $1.04 \times 10^{-6} \mathrm{sec}^{-1}$ and $2.08 \times 10^{-6} \mathrm{sec}^{-1}$. The deformation tests were performed at $1400^{\circ} \mathrm{C}$ in the air.

\subsection{TEM foil preparation}

TEM samples were prepared from the deformed samples. All the foils were cut parallel to the primary slip planes, $\{001\}$ for the sample deformed along $<112>$ in order to study the dislocation structures. Then the samples were polished, and cut into $3 \mathrm{~mm}$ disk, dimple polished and finally ion-bean thinning by following the conventional TEM sample preparation procedure.

\subsection{TEM observation}

The analysis of dislocation loops in this study was performed in Philips 400T, operating at $125 \mathrm{kv}$. Conventional diffraction contrast experiments using dynamical twobeam conditions ${ }^{5)}$ were carried out to determine the Burgers vector and study the dislocation structures and interaction. In theses two beam conditions, each of the Burgers vector can be determined from $g \cdot b$ analysis, with at least two $g \cdot b$ conditions.

\section{Results and Discussion}

$\mathrm{ZrO}_{2}$ exhibits plastic deformation by the activation of dislocation movements at high temperatures. The plastic behavior of this crystal changed by increasing strain rate from $1.04 \times 10^{-6} \mathrm{sec}^{-1}$ to $2.08 \times 10^{-6} \mathrm{sec}^{-1}$ by showing higher Young's modulus and yield stress, as shown in Fig. 2. And yield drop was also suppressed by increasing strain rate. And after yield point, work hardening rate was also

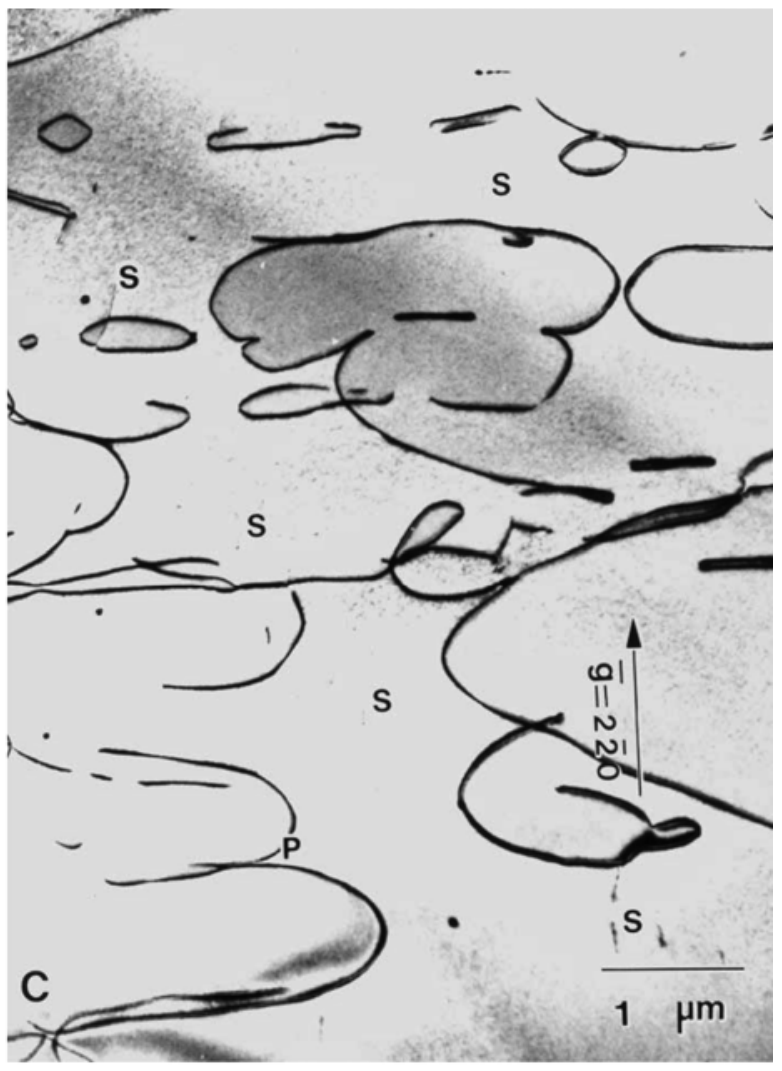

Fig. 3. Dislocation structures of the sample deformed to stage $\mathrm{C}_{4}$, showing edge type dislocation dipoles and loops on (001) plane. The secondary dislocations (Marked by $\mathrm{S}$ ) were out of contrast.

increased with increasing strain rate. Ingel at. al. ${ }^{6}$ reported that the yield drop became smaller as increasing strain rate from $3.0 \times 10^{-5}$ to $6.2 \times 10^{-4} \mathrm{sec}^{-1}$. The sample deformed to the elastic limit with strain rate of $1.04 \times 10^{-6} \mathrm{sec}^{-1}$ exhibited many dislocation dipoles and loops as shown in Fig. 3.

In fluorite crystals, the Burgers vector of dislocation is always $1 / 2<110>$ which is the shortest lattice vector connecting like ions and the primary slip system is $\{100\}$ $<110>$. ${ }^{7}$ These dipoles and prismatic loops were edge type and had the same Burgers vector as the primary dislocations. In the early stage of deformation, dislocation dipoles play important role in this crystal. The formation of dipoles can be caused by either dislocation-impurity interactions ${ }^{8)}$ or dislocation-dislocation interaction. ${ }^{9}{ }^{10)}$ Gilman et. al. ${ }^{8)}$ proposed that screw dislocations were pinned by jogs. Then those screw dislocations were dragged by those jogs and edge type dipoles were formed. This type of dislocation dipoles was also observed in many materials such as $\mathrm{LiF}^{8)} \mathrm{UO}_{2}{ }^{11)}$ and Y-CSZ crystal. ${ }^{1,2)}$ When two edge type dislocations of opposite Burgers vectors 


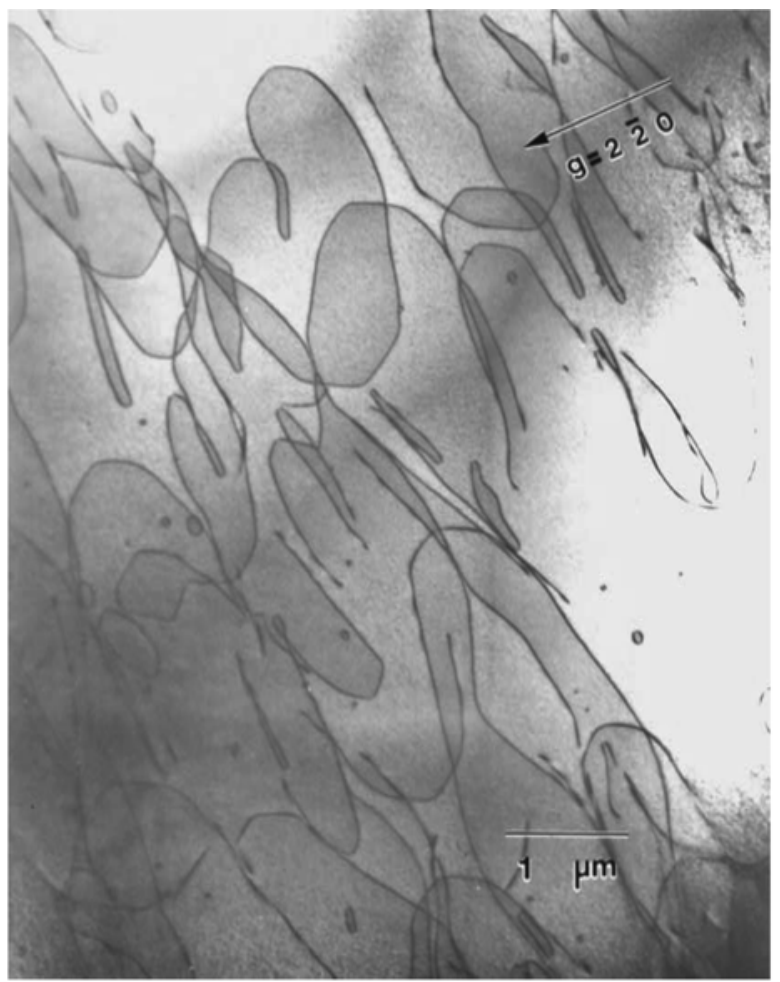

Fig. 4. Dislocation structures of the sample deformed to stage $\mathrm{A}_{41}$, showing many edge type dislocation dipoles and small prismatic loops on (001) plane.

are approaching each other on nearby parallel slip planes, they trapped each other, which is called edge trapping mechanism. ${ }^{8)}$ At the same time, the screw portions of dislocation also trapped each other and these can be annihilated easily through cross slip, leaving dislocation dipoles. ${ }^{8)}$ This type of dipoles were observed in the basal plane of HCP crystals, such as Mg, ${ }^{9)}$ and Sapphire. ${ }^{12)}$ The dipoles can be pinched off break into loops by dislocation climb $^{13)}$ or cross slip ${ }^{10)}$ due to the reduction of dislocation line energy. Since these dipoles were mostly edge in character, the resulting loops were prismatic loops, ether vacancy or interstitial type. Elastic strain fields around these dipoles and prismatic loops interrupt the movements of other dislocations resulting in work hardening in the early stage of plastic deformation of this crystal. And few secondary dislocations (marked by $\mathrm{S}$ ) were observed at this stage. With increasing strain rate from $1.04 \times 10^{-6} \mathrm{sec}^{-1}$ to $2.08 \times 10^{-6} \mathrm{sec}^{-1}$, there were no appreciable change of dislocation structures by showing edge-type dipole and prismatic loops, as shown in Fig. 4. It indicates that the early stage of plastic deformation of the crystal also involves formation of edge-type dipoles and prismatic loops in both samples. The difference in the dislocation

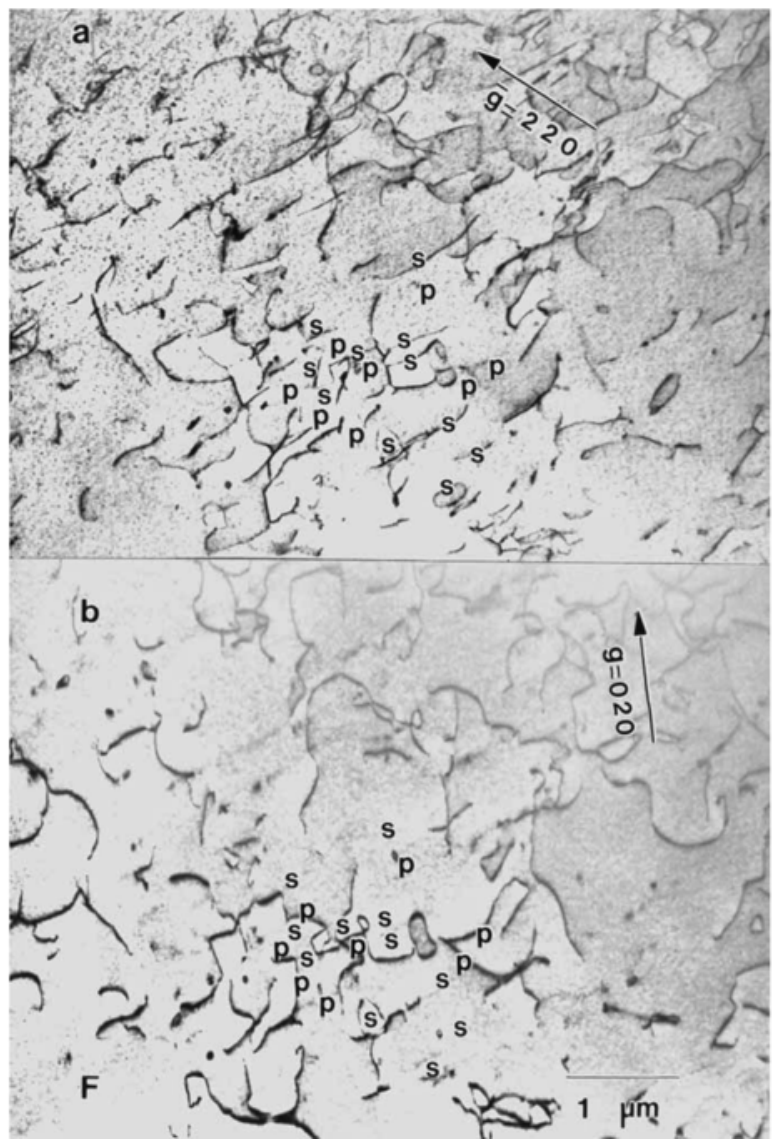

Fig. 5. Dislocation structures of the sample deformed to stage $\mathrm{F}_{4}, \mathrm{~g} \cdot \mathrm{b}=0$ for the primary dislocation in the upper micrograph, while the secondary dislocations were out of contrast in the lower micrograph.

structures was that the density of dislocation was higher and dislocation dipoles and loops were longer than those of the sample deformed at the lower strain rate, as shown in Fig. 3 and 4. The higher dislocation density by increasing strain rate can be explained by the Orowan equation, i.e. the linear relationship between strain rate and dislocation density,

$$
\varepsilon=\mathrm{b} \cdot \mathrm{N} \cdot \mathrm{v}
$$

Where $\varepsilon$ is strain rate, $\mathrm{b}, \mathrm{N}$ and $\mathrm{v}$ are Burgers vector, dislocation density and dislocation velocity, respectively. Higher dislocation density could result from the fact that the dislocation multiplication rate was higher than the recovery rate. The relationship between dislocation density and stress can be explained by the following equation,

$$
\tau_{\mathrm{a}}=\mathrm{c} \cdot \mathrm{G} \cdot \mathrm{b}\left(\mathrm{N}_{\mathrm{t}}\right)^{1 / 2}+\left(\varepsilon / \mathrm{A} \cdot \mathrm{b} \cdot \mathrm{N}_{\mathrm{m}}\right)^{-1 / 2}
$$

Where $\tau_{\mathrm{a}}, \mathrm{G}$, and are the applied stress and shear stress. A and $\mathrm{c}$ are constants. And $\mathrm{N}_{\mathrm{t}}$ and $\mathrm{N}_{\mathrm{m}}$ are total and mobile 


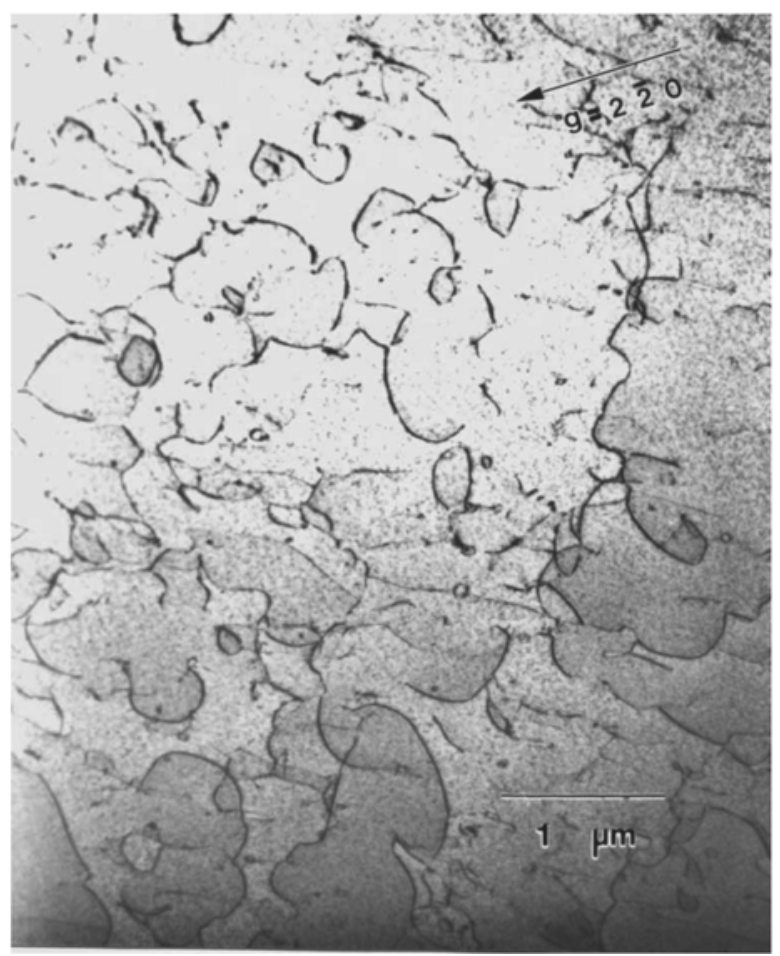

Fig. 6. Dislocation structures of the sample deformed to stage $\mathrm{B}_{41}$, which showed the dislocation tangles and networks which were formed by dislocation reactions in many slip systems.

dislocation density, respectively. The first term corresponds to the internal stress and the secondary term is the effective stress. The higher dislocation density and higher strain rate increase the effective stress. It may also cause the absence of yield drop at higher strain rate. However, there were no secondary dislocations in the sample deformed with higher strain rate. It may indicates that yield drop was very closely related to the activation of secondary slip system. Cheong at. al. ${ }^{2)}$ reported that yield drop was caused by activation of dislocations in the secondary slip system.

As the plastic deformation proceeded further into the plateau region, the dislocation structure became similar to that in the zero work hardening region of the sample deformed at lower strain rate. At lower strain rate, the density of secondary dislocations was increased remarkably and comparable to the density of the primary dislocations as shown in Fig. 5. The upper micrograph shows the dislocation structure when the primary dislocations (Marked by $\mathrm{P}$ ) were absent at stage $\mathrm{F}_{4}$. And the lower micrograph shows the dislocation structure of the same position of the upper micrograph at which the secondary dislocations (Marked by S) were out of contrast. It may indicates that in this region of plastic deformation, the dislocation in new slip system induced the recovery of stress by

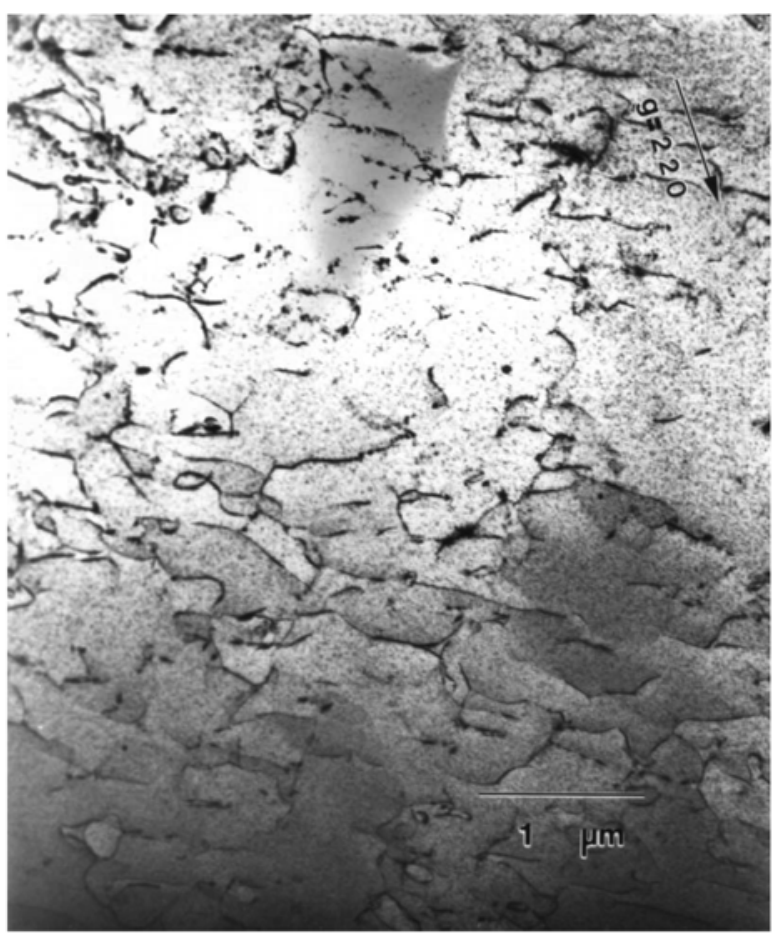

Fig. 7. Dislocation structures of the sample deformed to stage $\mathrm{B}_{41}$, The micrograph showed the dislocation structures when the primary dislocations were absence with $g \cdot b=0$.

providing more mobile dislocations. It was suggested that the interactions of the primary dislocations did not change the mobility of the secondary dislocations. ${ }^{14)}$ It may explain the zero work hardening region in the later stage of plastic deformation of this sample. With increasing strain rate, there was no appreciable change of dislocation structures. Fig. 6 shows the dislocation structure of the sample at the stage, $\mathrm{B}_{41}$. The dislocation structure was similar to one of the lower stain rate. Fig. 7 shows the dislocation structure when the primary dislocations were out of contrast, The secondary dislocation structure was similar to that of the primary dislocation which showed dislocation nodes, tangles and finally dislocation networks. The dislocation networks were formed by dislocation reactions in many slip systems. All six $<110\rangle$ type Burgers vectors were observed at this stage. Thus, double cross slip mechanism and formation of dislocation networks were insensitive to the change of strain rate.

As plastic deformation progressed to stage $\mathrm{G}_{4}$, density of dislocations were increased and the dislocations with many slip systems were operating through the convention dislocation multiplication. ${ }^{15)}$ At this stage this sample showed dislocation networks with many dislocation nods which caused work-hardening at the stage $\mathrm{G}_{4}$. The dis- 
location network and nods indicate that multiple slip system was activated. More dislocations in other slip system than those of the primary slip system were observed in the later stage of deformation.

\section{Conclusion}

Y-CSZ singe crystal showed plastic deformation at $1400^{\circ} \mathrm{C}$. When the strain rate rose from $1.04 \times 10^{-6} \mathrm{sec}^{-1}$ to $2.08 \times 10^{-6} \mathrm{sec}^{-1}$, Young's modulus and yield stress were increased. However, yield drop was suppressed by increasing the strain rate. The higher strain rate also increased the density of dislocation, which can be explained by the Orowan relation. The zero work hardening region can be caused by the fact that the dislocation multiplication rate was higher than the recovery rate. There was no appreciable change of dislocation structure which showed dipoles and loops in the early stage, nodes, tangles and networks in the later stages of deformation by increasing strain rate.

\section{Acknowledgement}

We are grateful for the financial support by the Korea Energy Management Corporation under grant 2008-N-FC12J-03-3-040.

\section{References}

1. E. Fries, F. Guiberteau, A. Dominguez-Rodriguez, D. -S. Cheong and A. H. Heuer, Phil. Mag. A, 60(1), 107 (1989).

2. A. Dominguez-Rodriguez, D. -S. Cheong and A. H. Heuer, Phil. Mag. A, 64(4), 923 (1991).

3. D. -S. Cheong, A. Dominguez-Rodriguez and A. H. Heuer, Phil. Mag. A, 60(1), 123 (1989).

4. W. G. Johnston, J. Appl. Phys., 33(9), 2050 (1962).

5. P. B. Hirsch, A. Howie, R. B. Nicholson, D. W. Pashley and M. J. Whelan, Electron Microscopy of Thin Crystals', Robert E. Krieger Pub. Co., Huntington, N.Y., USA (1977).

6. R. P. Ingel, D. Lewis, B. A. Bauer and R. W. Rice, J. Am. Ceram. Soc., 65(108), 1203 (1982).

7. S. Amelincx and W. J. Dekeyser, J. Appl. Phys., 29, 1000 (1958).

8. J. J. Gilman and W. G. Johnston, Solid State Physics, 13, (1963).

9. P. B. Hirsch and T. E. Mitchell, Work Hardening of Metals, ed. J. P. Hirth and J. Weertman, Gordon and Beach Science Publishers, Inc., N. Y., USA (1968).

10. A. S. Tetelman, Acta. Met., 10(9), 813 (1962).

11. C. S. Yust and C. J. MaHargue, J. Nucl. Mat. 31, 121 (1969).

12. B. J. Pletka, T. E. Mitchell and A. H. Heuer, J. Am. Ceram. Soc., 57(9), 388 (1974).

13. R. L. Segall, P. G. Partridge, and P. B. Hirsch, Phil. Mag., 6, 1493 (1961).

14. D. -S. Cheong, Ph. D. Thesis, C. W. R. U., Cleveland, Ohio (1989).

15. J. J. Gilman and W. G. Johnston, Dislocations and Mechanical Properties of Crystals, p.116, Wiley, N.Y., USA (1956). 